\title{
ON AN INTEGRAL OF LOMMEL AND BESSEL FUNCTIONS
}

\author{
M. ASLAM CHAUDHRY ${ }^{1}$
}

(Received 7 October 1991; revised 24 April 1992)

\begin{abstract}
In this paper we have evaluated an infinite integral of product of the Lommel and Bessel functions and powers. Some special cases of the result are discussed.
\end{abstract}

\section{Introduction}

The Struve functions and Neumann and Schlafli polynomials are special cases of the Lommel functions $s_{\mu, \nu}$ [10, pages 228-351]. The Lommel functions are particular integrals of the inhomogeneous Bessel differential equation

$$
z^{2} \frac{d^{2} w}{d z^{2}}+z \frac{d w}{d z}+\left(z^{2}-v^{2}\right) w=z^{\mu+1}
$$

where $\mu$ and $\nu$ are constants. These functions are encountered in the analytic theory of light [9, pages 392-395], the theory of diffraction [10, page 337], acoustical problems associated with determination of fluid pressure on a vibrating disk and, in the theory of loud speaker diaphragms [7, page 76], [6]. It should be noted that few results of integrals involving products of Bessel and Struve functions exist in the literature (see $[4,8,10]$ ). These integrals arise in the solutions to dual integral equations in which the kernel is the Bessel function. See [5, pages 201-204] and [1, pages 404-406]. For example, it can be seen that [5, page 203] $g(y)=(-1)^{n} \sqrt{2} \frac{\Gamma(n+1)}{\Gamma\left(n+\frac{1}{2}\right)} y^{-\frac{1}{2}} H_{\left(n+\frac{1}{2}\right)}(y), n \geq 0$, solves the dual

\footnotetext{
'Dept of Math. Sciences, King Fahd Univ. of Petroleum and Minerals, Dhahran, Saudi Arabia. (C) Australian Mathematical Society, 1994, Serial-fee code 0334-2700/94
} 
integral equations

$$
\begin{array}{ll}
\int_{0}^{\infty} g(y) J_{n}(x y) d y=x^{n}, & 0 \leq x<1 \\
\int_{0}^{\infty} y g(y) J_{n}(x y) d y=0, & 1 \leq x<\infty .
\end{array}
$$

The integral equations arise in the problems of finding the potential field in different types of practical problems. See [1, page 404] and [5, page 203]. In this paper we have solved an infinite integral of product of the Lommel and Bessel functions and powers. Some special cases of the result involving products of Bessel and Struve functions are discussed.

THEOREM 1.

$$
\begin{aligned}
\int_{0}^{\infty} t^{\nu-k} J_{v+k}(2 p t) s_{2 k, 2 \mu}(2 a t) d t & \\
= & 2^{2 k-3} a^{2 \mu} \frac{\Gamma\left(\mu+v+\frac{1}{2}\right) \Gamma\left(v-\mu+\frac{1}{2}\right)}{\Gamma(v-k+1) p^{2 \mu+\nu-k+1}} \\
& \times{ }_{2} F_{1}\left(\mu+v+\frac{1}{2}, \mu-k+\frac{1}{2} ; v-k+1 ; \frac{p^{2}-a^{2}}{p^{2}}\right) \\
(\operatorname{Re}(k \pm \mu)> & \left.-\frac{1}{2}, \operatorname{Re}(v+k)>-1, \operatorname{Re}(v \pm \mu)>-\frac{1}{2}, \operatorname{Re} p>0\right) .
\end{aligned}
$$

Proof. Let us define

$$
f(t)=t^{k} \exp \left(\frac{a}{2 t}\right) W_{k, \mu}\left(\frac{a}{t}\right), \quad \arg (a)<\pi,
$$

where $W_{k, \mu}$ is the Whittaker function. Then, according to [3, page 217(21)]

$$
\begin{aligned}
F(x)= & L\{f(t) ; x\}=2^{1-2 k} a^{\frac{1}{2}} x^{-k-\frac{1}{2}} s_{2 k, 2 \mu}(2 \sqrt{a x}) \\
& \left(\operatorname{Re}(k \pm \mu)>-\frac{1}{2}, \operatorname{Re}(p)>0\right) .
\end{aligned}
$$

Replacing $t$ by $1 / t$ in (3) and then multiplying both sides by $t^{\nu+k-1}$, we get

$$
t^{\nu+k-1} f(1 / t)=t^{\nu+1} \exp \left(\frac{a}{2} t\right) W_{k, \mu}(a t) .
$$

Using the result [3, page $216(16)]$, we have

$$
\begin{aligned}
L\left\{t^{\nu+k-1} f(1 / t) ; p\right\}= & \frac{\Gamma\left(\mu+\nu+\frac{1}{2}\right) \Gamma\left(\nu-\mu+\frac{1}{2}\right) a^{\mu+\frac{1}{2}}}{2 \Gamma(\nu-k+1) p^{\nu+\mu+\frac{1}{2}}} \\
& \times{ }_{2} F_{1}\left(\mu+\nu+\frac{1}{2}, \mu-k+\frac{1}{2} ; \nu-k+1 ; \frac{p-a}{p}\right) \\
& \left(\operatorname{Re}(\nu \pm \mu)>-\frac{1}{2}, \operatorname{Re} p>0\right) .
\end{aligned}
$$


However, according to [3, page 132(25)]

$$
\begin{aligned}
L\left\{t^{\nu+k-1}\right. & f(1 / t) ; p\}=p^{-(\nu+k) / 2} \int_{0}^{\infty} x^{(\nu+k) / 2} F(x) J_{v+k}(2 \sqrt{p x}) d x \\
& (\operatorname{Re}(\nu+k)>-1) .
\end{aligned}
$$

Equating (6) and (7) we get

$$
\begin{gathered}
\int_{0}^{\infty} x^{(v+k) / 2} F(x) J_{v+k}(2 \sqrt{p x}) d x \\
=\frac{\Gamma\left(\mu+v+\frac{1}{2}\right) \Gamma\left(v-\mu+\frac{1}{2}\right) a^{\mu+\frac{1}{2}}}{2 \Gamma(v-k+1) p^{(v+2 \mu-k+1) / 2}} \\
\times_{2} F_{1}\left(\mu+v+\frac{1}{2}, \mu-k+\frac{1}{2} ; v-k+1 ; \frac{p-a}{p}\right) \\
\left(\operatorname{Re}(k \pm \mu)>-\frac{1}{2}, \operatorname{Re}(v+k)>-1, \operatorname{Re}(v \pm \mu)>-\frac{1}{2}, \operatorname{Re} p>0\right) .
\end{gathered}
$$

Substituting the value of $F(x)$ in (8) we get

$$
\begin{aligned}
\int_{0}^{\infty} x^{(v-k-1) / 2} J_{v+k}(2 \sqrt{p x}) s_{2 k, 2 \mu}(2 \sqrt{a x}) d x \\
=2^{2 k-2} \frac{\Gamma\left(\mu+v+\frac{1}{2}\right) \Gamma\left(v-\mu+\frac{1}{2}\right) a^{\mu}}{\Gamma(v-k+1) p^{(v+2 \mu-k+1) / 2}} \\
\quad{ }_{2} F_{1}\left(\mu+v+\frac{1}{2}, \mu-k+\frac{1}{2} ; v-k+1 ; \frac{p-a}{p}\right) .
\end{aligned}
$$

Replacing $a$ by $a^{2}$ and $p$ by $p^{2}$ in (9) we get

$$
\begin{aligned}
\int_{0}^{\infty} x^{(\nu-k) / 2} J_{v+k}(2 p \sqrt{x}) s_{2 k, 2 \mu}(2 a \sqrt{x}) \frac{d x}{2 \sqrt{x}} \\
=2^{2 k-3} \frac{\Gamma\left(\mu+\nu+\frac{1}{2}\right) \Gamma\left(\nu-\mu+\frac{1}{2}\right) a^{2 \mu}}{\Gamma(\nu-k+1) p^{\nu+2 \mu-k+1}} \\
\quad{ }_{2} F_{1}\left(\mu+v+\frac{1}{2}, \mu-k+\frac{1}{2} ; v-k+1 ; \frac{p^{2}-a^{2}}{p^{2}}\right) .
\end{aligned}
$$

The transformation $t=x^{2}$ in (10) yields the proof of the theorem.

\section{COROLlaRY 1.}

$$
\begin{gathered}
\int_{0}^{\infty} x^{\nu-k} J_{\nu+k}(2 p t) s_{2 k, 2 \mu}(2 p t) d t=2^{2 \mu-3} \frac{\Gamma\left(\mu+\nu+\frac{1}{2}\right) \Gamma\left(\nu-\mu+\frac{1}{2}\right)}{\Gamma(\nu-k+1) p^{\nu-k+1}} \\
\left(\operatorname{Re}(k \pm \mu)>-\frac{1}{2}, \operatorname{Re}(\nu \pm \mu)>-\frac{1}{2}, \operatorname{Re}(\nu+k)>-1, \operatorname{Re} p>0\right) .
\end{gathered}
$$


PROOF. This follows from the theorem when we take $p=a$. In particular when $k=\mu$ in (11) we get

$$
\begin{gathered}
\int_{0}^{\infty} t^{\nu-\mu} J_{v+\mu}(2 p t) H_{2 \mu}(2 p t) d t=4^{-1} \pi^{-\frac{1}{2}} \frac{\Gamma\left(\mu+\nu+\frac{1}{2}\right) \Gamma\left(\nu-\mu+\frac{1}{2}\right)}{\Gamma(\nu-\mu+1) \Gamma\left(2 \mu+\frac{1}{2}\right)} p^{\mu-\nu-1} \\
(\operatorname{Re} \mu>-1 / 4, \operatorname{Re}(\nu \pm \mu)>-1, \operatorname{Re} p>0)
\end{gathered}
$$

COROLLARY 2.

$$
\int_{0}^{\infty} J_{v}(b t) H_{v}(b t) d t=\frac{1}{2 b} \quad(\operatorname{Re} v>-1, \quad \operatorname{Re} b>0) .
$$

Proof. This follows from (12) when we take $\mu=v$ and put $2 p=b$.

REMARK 1. It should be noted that (13) is an interesting identity because we also have [4, page $665(6.511)(1)]$

$$
\int_{0}^{\infty} J_{v}(b t) d t=\frac{1}{b} \quad(\operatorname{Re} b>0, \operatorname{Re} v>-1) .
$$

REMARK 2. It is interesting to see that (13) can be proved directly for $v=0$ as follows. According to [7, page 207],

$$
H_{0}(z)=\frac{4}{\pi} \sum_{n=0}^{\infty} \frac{J_{2 n+1}(z)}{2 n+1}
$$

Multiplying both sides of (14) by $J_{0}(z)$ and integrating from 0 to $\infty$, we get

$$
\int_{0}^{\infty} J_{0}(z) H_{0}(z) d z=\frac{4}{\pi} \sum_{n=0}^{\infty} \int_{n=0}^{\infty} \frac{1}{2 n+1} \int_{0}^{\infty} J_{0}(z) J_{2 n+1}(z) d z .
$$

However, according to [4, page 666(6.512)(2)],

$$
\int_{0}^{\infty} J_{0}(z) J_{2 n+1}(z) d z=\frac{(-1)^{n}}{2}
$$

and substitution of $x=\pi / 2$ in the Fourier sine series

$$
x=2\left(\frac{\sin x}{1}-\frac{\sin 2 x}{2}+\frac{\sin 3 x}{3}-\frac{\sin 4 x}{4}+\cdots\right), \quad-\pi<x<\pi
$$

yields

$$
\frac{\pi}{4}=\sum_{n=0}^{\infty} \frac{(-1)^{n}}{2 n+1}
$$

From (15) - (17) we get the proof of (13) for $v=0$. 
COROLlaRY 3. (see [4, page 405(3.721)]).

$$
\int_{0}^{\infty} \frac{\sin (a t)}{t} d t=\frac{\pi}{2} \quad(\operatorname{Re} a>0)
$$

PROOF. Substituting $v=-\frac{1}{2}$ in (13) and using the relation $H_{-\frac{1}{2}}(z)=J_{\frac{1}{2}}(z)$ we get

$$
\int_{0}^{\infty} J_{-\frac{1}{2}}(b t) J_{\frac{1}{2}}(b t) d t=\frac{1}{2 b} \text {. }
$$

The substitution of the values of $J_{-\frac{1}{2}}(b t)$ and $J_{\frac{1}{2}}(b t)$ from [4, page 966(8.464)] into (19) yields the proof of (18).

COROLlaRY 4.

$$
\begin{aligned}
& \int_{0}^{\infty} J_{v+k}(2 p t) G_{13}^{31}\left(p^{2} t^{2} \mid \begin{array}{l}
\frac{1}{2}+\frac{v}{2}+\frac{k}{2} \\
\frac{1}{2}+\frac{v}{2}+\frac{k}{2}, \frac{v}{2}+\mu-\frac{k}{2}, \frac{v}{2}-\mu-\frac{k}{2}
\end{array}\right) d t \\
& \quad=\left[\frac{\Gamma\left(\mu+v+\frac{1}{2}\right) \Gamma\left(v-\mu+\frac{1}{2}\right)}{p \Gamma(v-k+1)}\right]\left[\frac{\Gamma\left(\frac{1}{2}-k-\mu\right) \Gamma\left(\frac{1}{2}-k+\mu\right)}{4^{1+k-\mu}}\right] \\
& \left(\operatorname{Re}(k \pm \mu)>-\frac{1}{2}, \operatorname{Re}(v \pm \mu)>-\frac{1}{2}, \operatorname{Re}(v+k)>-1, \operatorname{Re} p>0\right) .
\end{aligned}
$$

PROOF. This follows from (11) when we use the facts [2, page 209(8)] and [3, page 220(55)].

COROLLARY 5.

$$
\begin{aligned}
\int_{0}^{\infty} t^{\nu-\mu} J_{v+\mu}(2 p t) H_{2 \mu}(2 a t) d t & \\
= & 4^{-1} \pi^{-\frac{1}{2}} \frac{\Gamma\left(\mu+v+\frac{1}{2}\right) \Gamma\left(\nu-\mu+\frac{1}{2}\right)}{\Gamma(v-\mu+1) \Gamma\left(2 \mu+\frac{1}{2}\right)} \frac{a^{2 \mu}}{p^{v+\mu+1}} \\
& \times_{2} F_{1}\left(\mu+v+\frac{1}{2}, \frac{1}{2} ; v-\mu+1 ; \frac{p^{2}-a^{2}}{p^{2}}\right) \\
(\operatorname{Re} \mu> & \left.-\frac{1}{4}, \operatorname{Re}(v \pm \mu)>-1, \operatorname{Re} p>0, \arg a<\pi\right) .
\end{aligned}
$$

Proof. This follows from the theorem when we taken $k=\mu$ and use the fact [4, page 986(8.573)(5)]. 


\section{Acknowledgements}

The author gratefully acknowledges the support provided for this research by the King Fahd University of Petroleum and Minerals. The referee's suggestions and helpful comments are appreciated.

\section{References}

[1] G. F. Carrier and C. E. Pearson, Functions of a complex variable (McGraw-Hill, 1966).

[2] A. Erdelyi et al., Higher transcendental functions, Volume I (McGraw-Hill Book Company, Inc., New York, 1954).

[3] A. Erdelyi et al., Tables of integral transforms, Volume I (McGraw-Hill Book Company, Inc., New York., 1954).

[4] I. S. Gradshteyn and I. M. Ryzhik, Tables of integrals, series and products (Academic Press, New York, 1980).

[5] J. D. Jackson, Classical electrodynamics, 2nd ed. (John Wiley \& Sons, 1975).

[6] N. W. McLachlan, "Sound pressure at any point on vibrating disk", Phil. Mag. 14 (1932) 1012.

[7] N. W. McLachlan, Bessel functions for engineers, 2nd ed. (Oxford, The University Press, 1955).

[8] N. W. McLachlan and A. L. Mayers, "Integrals involving Bessel and Struve functions", Phil. Mag. 21 (1936) 437-448.

[9] Siemon and J. Walker, The analytic theory of light (Cambridge, New York, 1904).

[10] G. N. Watson, A treatise on the theory of Bessel functions (Cambridge, The University Press, New York, 1966). 